\title{
PRINCIPIO DE PROPORCIONALIDAD SISTÉMICO
}

\author{
Wilson Yesid Suárez Manrique \\ Magister en Hermenéutica Jurídica y Derecho de la Universidad Industrial de Santander, (Colombia). Especialista \\ en Derecho Procesal. Coord. Especialización en Derecho Constitucional, Universidad Santo Tomás Bucaramanga, \\ Docente Facultad de Derecho, Investigador, Universidad Santo Tomás Bucaramanga (Colombia). \\ Correo electrónico: wilsonyesidsuarez@gmail.com
}

\section{Resumen}

El objetivo principal del presente escrito consiste en trazar una perspectiva metodológica alterna que ofrezca una visión más sistemática del principio de proporcionalidad, mediante la cual se dé cuenta de la concepción del ordenamiento jurídico como un sistema normativo y de la realidad de la práctica judicial en el control de constitucionalidad de las leyes.

Palabras clave: Proporcionalidad, subprincipios, sistema, práctica judicial, libertad, igualdad, democracia, división de poderes.

\section{Abstract}

The main objective of this paper is to sketch an alternative methodological perspective that offers a more systematic view of the proportionality principle that accounts for the conception of law as a normative system and reality of judicial practice in controlling constitutionality of laws.

Key Words: Proportionality, subbeginning, system, judicial practice, freedom, equality, democracy, division of power.

\section{Résumé}

L'objectif principal du présentécrit consiste à tracer une perspective méthodologique alternative qui offre une vision plus systématique du principe de proportionnalité, aumoyen de laquelleil se rendcompte de la conception de l'ordonnance juridique comme d'un système normatif et de la réalité de la pratique judiciaire dans le contrôle de constitutionnalité des lois.

Mots-clés: Une proportionnalité, des sous-principes, un système, une pratiquejudiciaire, une liberté, une égalité, une démocratie, une division de pouvoirs. 

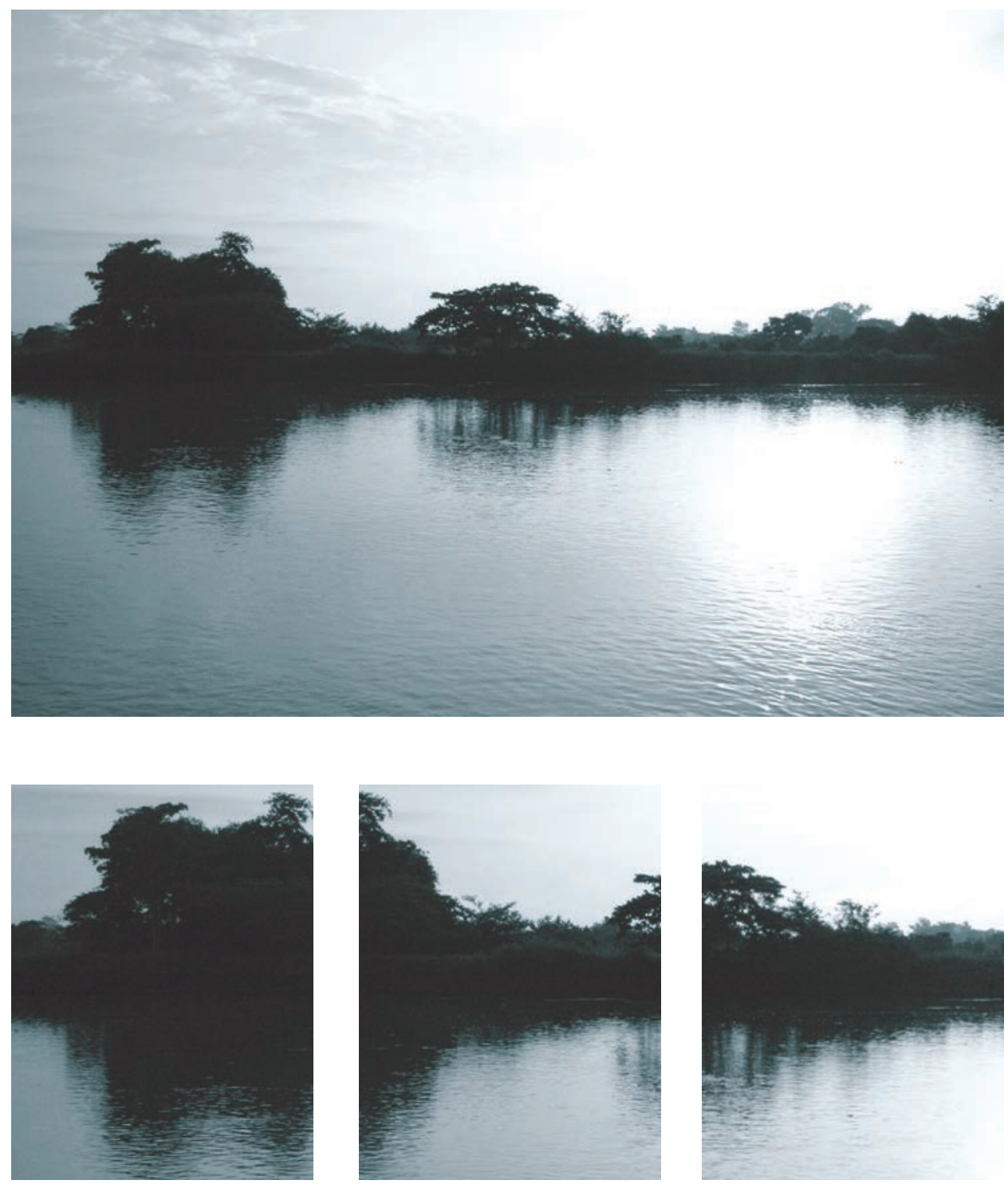

Rio Magdalena - Mompóx - Colombia

Martín Emilio Hernández Manrique 


\section{PRINCIPIO DE PROPORCIONALIDAD SISTÉMICO*}

Wilson Yesid Suárez Manrique

\section{Proemio}

Desde sus inicios y durante su vigencia el ordenamiento jurídico colombiano se ha caracterizado por el flujo y reflujo en la recepción de teorías jurídicas provenientes de sitios centrales de producción. Es así, que pueden observarse los paradigmas formalista, antiformalista, kelseniano y moderno, en etapas, más o menos, secuenciales en la conformación evolutiva del sistema jurídico colombiano (López, 2004).

Con el cambio de paradigma implantado por la Constitución de 1991, se ha dado un gran paso para desarraigar el siempre recurrente clasismo que caracteriza el ordenamiento jurídico. Son varios los ítems en los cuales puede notarse los principales puntos de cambio del actual paradigma de derecho. Entre ellos se destacan: el contenido material y normativo de la Constitución, su relación con la moral, la modificación del sistema de fuentes, la descodificación del derecho, y, para el caso, la forma de interpretar y aplicar el derecho.

La forma "normal" de la aplicación del derecho en el ordenamiento jurídico colombiano, de conformidad con su linaje romano-germano, ha sido la subsunción. Se entendía la norma como un enunciado hipotético, el cual estaba conformado por un presupuesto de hecho y una consecuencia jurídica. Los hechos se subsumían en el presupuesto de hecho y la decisión era la consecuencia jurídica.

Con el advenimiento del nuevo paradigma constitucional, esta forma de entender la metodología jurídica varió. Principalmente, por el entendido axiológico, sistémico y finalista de la Constitución, y por la estructura de los principios constitucionales.

* $\quad$ El texto aquí presentado es producto del proyecto Interpretación Contractual Adecuada, que adelanta el autor. Quinta Convocatoria Interna. 2010. Grupo de Investigación Neoconstitucionalismo y Derecho. Facultad de Derecho. Universidad Santo Tomás Bucaramanga - Colombia. 
Al variarse la naturaleza y estructura de la formulación jurídica ha de entenderse que debió variar la forma de aplicarla. La subsunción se vio sobrepasada por metodologías más elaboradas como los test de ponderación, armonización o el principio de proporcionalidad (Bernal, 2009; 2007).

Esta nueva metodología jurídica no fue, en principio, creación de la jurisprudencia o doctrina nacional (López, 2006). Se trató, en el mayor de los casos, de la recepción de metodologías elaboradas en sitios de producción de países centrales. En el caso de la metodología jurídica colombiana, se han tenido importantes aportes de la doctrina y jurisprudencia germana y anglosajona.

La Corte Constitucional ha tomado tales desarrollos y ha planteado, de diversas formas, desde juicios de proporcionalidad "simples" hasta "integrados". La esencia del test de proporcionalidad utilizado por nuestra Corte Constitucional, en sus diferentes facetas, es una muestra de la adaptación de las teorías jurídicas foráneas para el sistema jurídico colombiano. Adaptación que trae consecuencias importantes para la comprensión de la Constitución y del rol de la Corte Constitucional.

A partir de la adopción que la Corte Constitucional efectuó del juicio de proporcionalidad y el test de igualdad, el objetivo principal del presente escrito consiste en bosquejar una perspectiva metodológica alterna que ofrezca una visión más sistemática del principio de proporcionalidad, que dé cuenta de la concepción del ordenamiento jurídico como un sistema normativo y de la realidad de la práctica judicial en el control de constitucionalidad de las leyes.

La principal objeción contra el principio de proporcionalidad a la cual se le desea dar respuesta es aquella que se ha denominado como "el reduccionismo del principio de proporcionalidad". Según esta objeción: 1) el principio de proporcionalidad es reduccionista de la concepción del ordenamiento jurídico como sistema, porque presupone que en la mayoría de las ocasiones, en los casos concretos, se enfrentan únicamente dos principios diametralmente opuestos, negando con esto la concepción sistémica del ordenamiento jurídico, según la cual existe una compleja relación entre los diversos principios, pues, la afectación (o el desarrollo) de un derecho fundamental (o principio) implica necesariamente la afectación, en diferentes grados, a otros derechos principios; y 2) la concepción clásica del principio de proporcionalidad no da cuenta de la práctica jurídica, por cuanto en el control de constitucional se puede evidenciar que la argumentación de la Corte Constitucional no se reduce a la confrontación de sólo dos principios, son amplios los ejemplos en los que en el control de constitucionalidad se consideran diversos principios en los dos lados de la ponderación.

Para cumplir la finalidad reseñada, el escrito se divide en tres partes. En la primera se bosquejan los elementos generales que soportan y describen el principio de proporcionalidad. En la segunda se señalan, brevemente, las principales objeciones efectuadas al principio de proporcionalidad, y la forma genérica en la cual éstas han tratado de ser salvadas o reducidas. En la tercera se plantea, principalmente, un bosquejo de la alternativa de la concepción clásica del principio de proporcionalidad, mediante el cual se entiende al ordenamiento jurídico como un sistema y se da cuenta de la práctica de la Corte Constitucional. 


\section{Elementos generales del principio de proporcionalidad}

\subsection{Estado Constitucional}

El tránsito del Estado de Derecho al Estado Constitucional implicó un cambio en la forma de entender el ordenamiento jurídico. La supremacía de la Constitución y la fuerza normativa de los principios constitucionales, trajeron consigo la necesidad de una nueva concepción en la metodología jurídica.

En el Estado Constitucional las leyes deben subordinarse materialmente a los postulados constitucionales, por tanto, se han creado o confeccionado diversos mecanismos que tienden a garantizar dicha conformidad. Uno de esos mecanismos es el de control de Constitucionalidad de la ley establecido en el artículo 241 de la Constitución Política. Para realizar este control de contenido material se deben contrastar los postulados constitucionales con las prescripciones de ley. La tarea de contrastar los postulados constitucionales con las leyes, tiene varios inconvenientes ontológicos y cognitivos; empero, para el caso de este escrito, se resalta, especialmente, la dificultad que se presenta para determinar el sentido y el alcance de los principios constitucionales, y el grado en que ellos pueden ser desarrollados, protegidos o restringidos.

El principio de proporcionalidad se erige como una herramienta metodológica para la interpretación y la aplicación de los principios constitucionales, que la Corte Constitucional ha adoptado para, ente otras cosas, efectuar el control de constitucionalidad de las leyes y la solución de conflictos entre derechos fundamentales.

El objetivo general de esta forma de concebir el control de constitucionalidad de las leyes es el de pretender garantizar la coherencia y consistencia en el ordenamiento jurídico. Lo cual, para el caso, implica, especialmente, la concepción del ordenamiento jurídico como un sistema normativo, de tipo jerárquico. La Corte Constitucional, en la sentencia C 037 de 2000, estableció:

"El ordenamiento jurídico colombiano supone una jerarquía normativa que emana de la propia Constitución.” (...). “(..) las normas constitucionales ocupan, sin discusión, el primer lugar dentro de la jerarquía del ordenamiento jurídico (...). Pero más allá de la supremacía constitucional, de la propia Carta también se desprende que las leyes expedidas por el Congreso dentro de la órbita de competencias que le asigna la Constitución, ocupan, en principio, una posición prevalente en la escala normativa frente al resto del ordenamiento jurídico.”(...). "La unidad del sistema jurídico, y su coherencia y armonía, dependen de la característica de ordenamiento de tipo jerárquico de que se reviste. La jerarquía de las normas hace que aquellas de rango superior, con la Carta Fundamental a la cabeza, sean la fuente de validez de las que les siguen en dicha escala jerárquica. Las de inferior categoría, deben resultar acordes con las superiores, y desarrollarlas en sus posibles aplicaciones de grado más particular. En esto consiste la connotación de sistema de que se reviste el ordenamiento, que garantiza su coherencia interna." 


\subsection{Principios y normas}

En los ordenamientos jurídicos modernos pueden rastrearse básicamente dos especies de reglas: las normas y los principios (Alexy, 2002; Bernal, 2009, 2007). Las normas dicen que algo debe hacerse, no hacerse o permitirse. Lo que dicen las normas son mandatos definitivos, pues, o se cumple o no se cumple. Tales normas suelen aplicarse mediante la subsunción. Por su parte, los principios son mandatos de optimización que ordenan que algo sea realizado en la mayor medida posible, dentro de las posibilidades fácticas y jurídicas (Alexy, 2002; 2004). Por tanto, los principios no son mandatos definitivos, pues, ellos pueden ser cumplidos en diferentes medidas, por lo cual, pueden existir principios que hayan sido desarrollados en diferente medidas. Tales principios suelen aplicarse mediante el principio de proporcionalidad o la ponderación ${ }^{1}$.

\subsection{Presupuestos del test de proporcionalidad}

La aplicación del principio de proporcionalidad $\mathrm{y}$, especialmente, del subprincipio de proporcionalidad, presupone que se considere a los derechos fundamentales como principios. Cuando se interpretan como principios se entiende que los derechos fundamentales son mandatos de optimización; este mandato indica que el objeto de los derechos fundamentales debe realizarse en la mayor medida posible, de acuerdo con las posibilidades fácticas y jurídicas (Alexy, 2002).

El entender los derechos fundamentales como principios permite, en especial, resaltar tres características importantes. Permite, en primer lugar, comprender que los derechos fundamentales pueden ser desarrollados en diferentes medidas. Es decir, que lo que ellos mandan pueden cumplirse en menor o mayor grado. En segundo lugar, los principios son un imperativo a que se busque en todos los casos una mayor realización de los derechos fundamentales, por tanto, ello implica que se expanda en la medida más amplia su espacio de desarrollo y protección. En tercer lugar, la consideración de principios admite la limitación de los derechos fundamentales, por tanto, consiente, pues, que bajo ciertos supuestos los derechos fundamentales puedan ser restringidos en aras a satisfacer otros principios constitucionales ${ }^{2}$.

1 No obstante lo anterior, en donde se ha establecido una diferencia de la aplicación de las normas y principios, entre la subsunción y la ponderación, se hace necesario efectuar una precisión. Se cree conveniente precisar que la ponderación en sí no es un mecanismo de aplicación al caso concreto, sino que es un mecanismo para crear una norma particular de precedencia. Pues, luego de que se establece la norma particular de precedencia es que se efectúa una subsunción mediante la cual se resuelve el caso concreto. La ponderación es un elemento para fijar una norma jurídica universal: la premisa mayor; mediante la cual se resuelve el caso. La aplicación de la norma creada se efectúa mediante la subsunción. Así, pues, en últimas, normas y principios se aplican mediante la ponderación.

2 Según Robert Alexy "el principio de proporcionalidad se vuelve relevante si aceptamos que no existen derechos absolutos, sino que cada derecho se enfrenta a la posibilidad de ser limitado". Sí 
Las limitaciones a los derechos fundamentales según el principio de proporcionalidad, en las circunstancias concretas, conlleva a que se entienda que los derechos fundamentales ostentan diferentes contenidos: un contenido prima facie y un contenido definitivo. El contenido prima facie se compone de las prerrogativas directas y adscritas que pueden interpretarse de los derechos fundamentales en un sentido general. Por la amplitud de este contenido prima facie fácilmente puede entenderse que entre en colisión con otros principios. Cuando entra en colisión con otro $\mathrm{u}$ otros principios y se interpreta mediante el principio de proporcionalidad se obtiene el contenido definitivo del principio para el caso en concreto.

\subsection{Subprincipios del principio de proporcionalidad}

De forma general el test de proporcionalidad puede dividirse en tres subprincipios: idoneidad, necesidad y proporcionalidad en sentido estricto.

El subprincipio de idoneidad es teleológico, requiere que las intervenciones en los derechos fundamentales deban ser adecuadas para contribuir a la obtención de un fin constitucionalmente legítimo. Es decir, se condiciona la intervención a la existencia de un fin legítimo a la luz de la constitución y la existencia de adecuación de la medida para el cumplimiento del fin. Por tanto, este subprincipio se divide en dos partes: "la legitimidad constitucional del objetivo y la adecuación de la medida examinada" (Bernal, 2009, 124).

Según el subprincipio de necesidad, "toda medida de intervención en los derechos fundamentales debe ser la más benigna con el derecho intervenido, entre todas aquellas que revisten por lo menos la misma idoneidad para contribuir a alcanzar el objetivo propuesto". Es decir, que dentro de las diferentes medidas que pueden ser idóneas para cumplir la finalidad constitucional referida, la que se escoja debe ser la que menos afecte el principio examinado. Por tanto, también, este subrincipio se divide en dos partes: la idoneidad equivalente o mayor del medio alternativo, y el menor grado en que éste intervenga en el derecho fundamental (Bernal, 2009, 124).

De conformidad con el subprincipio de proporcionalidad en sentido estricto, "las ventajas que se obtienen mediante la intervención en el derecho fundamental deben compensar los sacrificios que ésta implica para sus titulares y para la sociedad en general" (Bernal, 2003, 36). Es decir, se deben comparar los beneficios de la intervención con sus inmolaciones. Luego, según la proporcionalidad en sentido estricto se permite que una satisfacción alta de un principio constitucional conlleve, prima facie, una afectación leve en otro.

Así pues, para que una intervención a un principio constitucional sea considerada como admisible, debe cumplir los requerimientos de los tres subprincipios señalados.

los derechos fundamentales se entendieran como mandatos absolutos, de carácter definitivo, que siempre deben ser aplicados, no tendrían ningún sentido tratar de sopesar su aplicación y establecer sus límites. 


\subsection{Proporcionalidad en sentido estricto}

La proporcionalidad en sentido estricto está conformada por tres elementos: la ley de la ponderación, la fórmula del peso, y las cargas de la argumentación. Según la ley de la ponderación: Cuando mayor sea el grado de no satisfacción o restricción de uno de los principios, tanto mayor deberá ser el grado de la importancia de la satisfacción del otro (Bernal, 2009, 124).

De lo anterior se infiere que la ley de la ponderación implica el desarrollo de tres etapas. En primera, la tarea se resume en determinar el grado de no satisfacción o restricción de uno de los principios. En la segunda, se establece la importancia de la satisfacción del principio contrario. Y en la tercera, "debe definirse si la importancia de la satisfacción del principio contrario justifica la restricción o la no satisfacción del otro"'s.

El segundo paso del principio de proporcionalidad en sentido estricto lo constituye la fórmula del peso. De forma general pueden establecerse dos versiones distintas, según se tenga predilección por una visión aritmética o geométrica de las magnitudes. Quien prefiera trabajar con magnitudes aritméticas desarrolla la formula diferencial" . "Sin embargo, la fórmula diferencial no da cuenta de una propiedad que resulta central para los principios". Si se toma como serie geométrica los valores $2^{\circ}, 2^{1}$ y $2^{2}$, es decir, 1,2 y 4 , se muestra como la diferencia de la serie aritmética, en esta serie, las distancias "respectivas entre los grados no son iguales sino que se incrementan". Lo cual representa "el hecho de que los principios ganan cada vez una fuerza mayor al aumentar la intensidad de la intervención, lo cual está en armonía con la tasa marginal decreciente de sustitución”.

Siguiendo a Bernal $(2005 ; 2009)$ la fórmula del peso geométrica es la siguiente:

$$
G P i, j C=-\left[-\frac{I P i C \cdot G P i A \cdot S P i C}{I P i C \cdot G P j A \cdot S P j C}\right.
$$

Donde: $\mathrm{I}=, \mathrm{P}=, \mathrm{C}=, \mathrm{G}=$....

Se espera que la aplicación de esta fórmula establezca, teniendo en cuenta las

3 Para poder llevar a cabo las tareas de las dos primeras etapas puede establecerse una escala triádica, según la cual puedan catalogarse la afectación y la importancia de la satisfacción con los grados "leve", "medio" y "grave", los cuales pueden ser caracterizados con las letras " $l$ ", " $m$ ” y " $g$ ”. Por ejemplo, en el caso citado la restricción de la libertad de profesión sería una interferencia leve mientras que la importancia de la protección de la satisfacción de la salud pública, seria intensa o grave.

4 La fórmula diferencial puede representarse de la siguiente forma: $G i, j=I i-I j$. Para aplicar esta fórmula los grados leve, medio y grave, referidos a continuación, pueden representarse mediante los números, 1, 2 y 3 respectivamente (Bernal, 2009). 
seis variables referidas 5 , la procedencia condicionada de un principio sobre otro. No obstante, no siempre ocurre así. En ocasiones se da un empate en la fórmula del peso, lo cual, da paso a la aplicación de la tercera etapa: las cargas de la argumentación. Esta tercera etapa de la proporcionalidad en sentido estricto opera cuando existe un empate en la aplicación de la fórmula del peso, es decir, cuando no ha podido establecerse la precedencia condicionada de un principio sobre el otro. Luego, "las cargas de la argumentación" constituyen la etapa para desempatar el peso concreto de los principios.

Sin embargo, pueden sostenerse, de forma general, dos perspectivas distintas sobre este asunto. La predilección por los principios en favor de la libertad e igualdad y la propensión por resolver los empates en favor del legislador. "Alexy parece defender dos diferentes formas de superar este tipo de empates, una en el capítulo final de la Teoría de los derechos fundamentales y otra en el Epílogo a esta obra." En el primer caso Alexy sugiere que el empate se debe resolver en favor de los principios de libertad y de igualdad. En el segundo caso sugiere que el empate se debe resolver en favor del legislador.

\subsection{La adopción del test de proporcionalidad}

En el ordenamiento jurídico colombiano la proporcionalidad como metodología de interpretación y aplicación de los principios constitucionales ha sido adoptada, principalmente, por la Corte Constitucional y algunos teóricos del Derecho. La Corte ha tratado de efectuar una adopción metodológica, en algunos casos, idiosincrática, tratando de combinarlo con poSIciones propias o con el test de igualdad de origen anglosajón ${ }^{6}$; por su parte, los teóricos del derecho, han tratado de tomar el principio y reconstruirlo en casos particulares y en diversas materias.

5 Las variables respectan a la importancia o afectación, el peso abstracto, y la estabilidad de las premisas empíricas de los dos principios en juego.

6 La sentencia de la Corte Constitucional C 093 de 2001, estableció: "La doctrina y la jurisprudencia constitucional comparadas, así como la propia práctica de esta Corporación, parecen indicar que existen dos grandes enfoques para analizar los casos relacionados con el derecho a la igualdad. El primero de ellos, que ha sido desarrollado principalmente por la Corte Europea de Derechos Humanos y por los tribunales constitucionales de España y Alemania, se basa en el llamado test o juicio de proporcionalidad, que comprende distintos pasos. Así, el juez estudia (i) si la medida es o no "adecuada", esto es, si ella constituye un medio idóneo para alcanzar un fin constitucionalmente válido; luego (ii) examina si el trato diferente es o no "necesario" o "indispensable", para lo cual debe el funcionario analizar si existe o no otra medida que sea menos onerosa, en términos del sacrificio de un derecho o un valor constitucional, y que tenga la virtud de alcanzar con la misma eficacia el fin propuesto. Y, (iii) finalmente, el juez realiza un análisis de "proporcionalidad en estricto sentido" para determinar si el trato desigual no sacrifica valores y principios constitucionales que tengan mayor relevancia que los alcanzados con la medida diferencial. La otra tendencia, con raíces en la jurisprudencia de la Corte Suprema de Estados Unidos, se funda en la existencia de distintos niveles de intensidad en los "escrutinios" o "tests" de igualdad (estrictos, intermedios o suaves). Así, cuando el test es estricto, el trato diferente debe constituir una medida necesaria para alcanzar un objetivo constitucionalmente imperioso, mientras que si el test es flexible o de mera razonabilidad, basta con que la medida sea potencialmente adecuada para alcanzar un propósito que no esté prohibido por el ordenamiento." 
La Corte Constitucional en algunas de sus sentencias ha aceptado y aplicado el principio de proporcionalidad como criterio metodológico de interpretación y aplicación de los derechos fundamentales. En la Sentencia C 093 de 2001 y 673 de 2001, reconoce la influencia de la doctrina jurídica alemana y de la jurisprudencia del Tribunal Europeo de Derechos Humanos, de la cual la Corte retoma las etapas que integran el juicio de proporcionalidad: (1) idoneidad para contribuir al logro de un fin constitucionalmente válido; (2) necesidad, entendida como la no existencia de un medio menos oneroso, en términos del sacrificio de otros principios constitucionales, para alcanzar el fin; y (3) proporcionalidad en sentido estricto7.

Bernal Pulido y Gloria Lopera, pueden considerarse como algunos de los autores más representativos en lo que concierne al tema de la revisión y reconstrucción del principio de proporcionalidad. Del primero se destaca su tesis doctoral sobre el Principio de Proporcionalidad y los derechos fundamentales, y los libros El Derecho de los derechos, El Neoconstitucionalismo a debate, y El Neoconstitucionalismo y la normatividad del derecho, donde se destacan algunos textos que de forma directa se dedican al estudio de dicho principio.

\section{CRÍTICAS A LA PONDERACIÓN}

\subsection{Tres objeciones generales}

Según los críticos la ponderación es irracional por una miscelánea de razones ${ }^{8}$.

$7 \quad$ Igualmente, se aprecia la influencia de la jurisprudencia estadounidense, en particular en el intento por establecer distintos niveles de intensidad en el escrutinio, en función del tipo de medida de cuya evaluación se trata (siendo más débil el escrutinio en aquellos ámbitos donde se reconoce una libertad de configuración más amplia al legislador), del criterio de diferenciación empleado (cuando se trata de medidas que comprometen el principio de igualdad) y del derecho fundamental en juego (en general para medidas que afectan la libertad de expresión se emplea un test estricto).

8 De forma general pueden dividirse, bajo otra perspectiva a que se presenta en el escrito, las críticas efectuadas, directa o indirectamente, contra la ponderación en tres grandes grupos: aquellas que atacan los presupuestos, las que atacan la metodología, y las que atacan las consecuencias. No obstante las complejas relaciones que entre ellas pueda existir. Las críticas que atacan los presupuestos de la ponderación están encaminadas a argumentar como los elementos que presupone la ponderación son problemáticos en sí mismos, por tanto, la ponderación no puede ser un elemento adecuado para la interpretación y aplicación de los derechos fundamentales. Se traen a colación cuatro críticas generales. La primera crítica acerca de la validez de la constitución "y su vinculación legislativa del ejecutivo y del judicial”. La segunda, cuestiona la misma existencia de los principios, bajo el argumento que no pueden diferenciarse claramente de las normas. La tercera, cuestiona que los principios digan algo, es decir, trata de enfatizar el carácter vacío de los principios. Las críticas que atacan la metodología de la ponderación están encaminadas a tratar de demostrar cómo no puede aplicarse adecuadamente el test. Se citan dos críticas generales. La primera refiere a que la ponderación es un juicio metodológico arbitrario y salomónico, es, en otras palabras, irracional. La segunda, muy relacionada con la categoría anterior, afirma que no se pueden ponderar los derechos porque son unidades inconmensurables. Las críticas que atacan las consecuencias de la ponderación están encaminadas a argumentar como la utilización de esa herramienta metodológica puede traer 
"Las más prominentes se refieren a la indeterminación de la ponderación, a la inconmensurabilidad a que se enfrenta su aplicación y a la imposibilidad de predecir sus resultados" (Bernal, 2009, 28).

Se dice que la ponderación es indeterminada por cuanto es un concepto ambiguo que no ostenta una estructura precisa y adecuada, por tanto, está lejos de ser un criterio metodológico que garantice la objetividad metodológica. Luego, en últimas, la ponderación permite que en la interpretación y aplicación de los principios constitucionales, se entremezclen una serie de elementos de la subjetividad del juez (Stammler, 1923, 447).

La inconmensurabilidad de la ponderación refiere a que las unidades que se pesan no son comparables, pues se ponderan unidades de naturaleza diferente, algo así como comparar peras con manzanas. Nada, desde este punto de vista, tiene que ver el derecho al honor y la libertad de expresión; pues, son unidades de naturaleza diferente que, por tanto, no pueden compararse.

La imprevisibilidad de los resultados alude a que no puede racionalmente predecirse los resultados de la ponderación en un caso concreto. Pues, cada juez que aplique la ponderación lo hará de tal manera que no puede saberse cuál es su resultado. Por consiguiente, "las decisiones judiciales que emergen de la ponderación conformarían una jurisprudencia ad hoc, que tendería a magnificar la justicia del caso concreto mientras, correlativamente, sacrificaría la certeza, la coherencia y la generalidad del derecho."

\subsection{El hiper-raccionalismo}

Pese que pueden aducirse razones de diferente tipo para tratar de salvar a la ponderación de estas objeciones, de forma genérica se ha expuesto por Bernal Pulido que tales objeciones son "hiper-racionalistas". Por cuanto, no reconocen que la racionalidad tiene ciertos límites. No puede buscarse una objetividad plena en el campo normativo o práctico general. Según esta forma de defensa la ponderación es, dentro de sus posibilidades, un método racional. Querer establecer la precisión en este campo como si se tratar de las ciencias matemáticas es hiperracionalismo. En este campo la razón tiene sus limitaciones pero ello no implica que el procedimiento de la ponderación sea irracional. El criticarle a la ponderación ser irracional por permitir cierto grado de subjetividad, es pasar por alto, que bajo dicho argumento, la subsunción, también sería irracional, pues, también permite cierto grado de discreción.

Este escrito no es el espacio adecuado para hacer un recuento o un balance pormenorizado de las relaciones entre las críticas, ni la forma, como éstas han tratado de ser refutadas. El objetivo es más modesto y con inclinaciones locales y

resultados inconvenientes. Se traen a colación tres críticas generales. La primera, dice que la aplicación de la ponderación conlleva a una supraconstitucionalización. La segunda manifiesta que la aplicación de la ponderación conlleva a la pérdida de la vigencia de los derechos fundamentales como normas. La tercera refiere a que los resultados de la ponderación son imprevisibles. 
prácticas. No pretende dar una solución ideal, sino un mecanismo de entendimiento de la ponderación acorde con la práctica jurídica colombiana. No pretende dar una solución definitiva, sino tratar de acercarse de manera hipotética a un mejor entendimiento de la práctica judicial. Para ello, se referirá de manera directa la crítica denominada como "reduccionismo de la ponderación". Crítica que pese a estar relacionada, con algunas de las críticas referidas, merece una especial atención por cuanto ha sido poco estudiada.

\subsection{Reduccionismo de la ponderación}

No obstante, las críticas efectuadas a la ponderación, en el presente escrito se quiere resaltar la crítica que afirma que el principio de proporcionalidad es reduccionista, por cuanto no refleja la concepción del ordenamiento jurídico como un sistema normativo ni la práctica jurídica acerca del control de constitucionalidad de las leyes.

De la exposición del principio de proporcionalidad y los ejemplos que se utilizan para ilustrarlo, se puede notar que existe un reduccionismo en la forma de entender el ordenamiento jurídico y la función judicial, pues, se muestra como en el caso concreto, en la mayoría de las ocasiones, se contraponen únicamente dos principios diametralmente opuestos. Es decir, se trata, por ejemplo, de honor vs. libertad de expresión; vida vs. derecho a libertad religiosa; libertad de cátedra vs. libertad religiosa. Se elimina, de este modo, la posibilidad de que en un ordenamiento jurídico, visto como un sistema, otros principios se vean afectados o satisfechos, tal como en realidad sucede. Si se considera el ordenamiento jurídico como un sistema se ha de entender que las relaciones existentes entre los diferentes elementos del sistema conllevan a que la afectación o satisfacción de uno de los elementos soporten la satisfacción o afectación de otros principios constitucionales. Por tanto, considerar que en un caso concreto sólo se contraponen dos principios constitucionales, presupone la negación de la consideración del ordenamiento jurídico como un sistema.

Asimismo, la práctica judicial del control de constitucionalidad de las leyes o de los actos legislativos, muestra como en el discurso de la Corte Constitucional, se revisa la contraposición, en la mayoría de los casos, de un conjunto de principios constitucionales en contra de otro grupo de principios constitucionales, es decir, que la práctica judicial en la utilización del principio de proporcionalidad no se limita a sopesar, de forma aislada, un principio con otro, sino un conjunto de principios.

\section{ALGUNAS IDEAS PARA LA SOLUCIÓN}

De forma general pueden revisarse la consideración de la fórmula del peso extendida de Alexy (Carbonell, 2008, 13-43), como un buen intento de solución de estos casos. No obstante, los problemas y vacíos que presenta hacen insalvable esa vía. Por lo cual, se hace necesario, revisar la manera como argumenta la Corte 
Constitucional, a efectos de sostener, así sea a manera de hipótesis, una solución a las necesidades referidas.

\subsection{La fórmula de peso extendida}

Robert Alexy considera de manera sumaria el evento en el cual en uno de los lados de la fórmula se encuentren varios principios. Y, trata de solucionar esta situación mediante lo que él denomina fórmula del peso extendida. De ella refiere dos versiones. La una puede ser llamada fórmula de peso extendida en sentido concreto y la otra puede ser llamada fórmula de peso extendida completa.

En la fórmula del peso extendida en sentido concreto el hecho de que existan varios principios en juego de uno de los lados de la ponderación se soluciona acumulando los principios de manera aditiva "sólo en el lado de la restricción". Luego, la fórmula sería la siguiente:

$$
\begin{aligned}
& I i \cdot G i \cdot S i \\
& G i, j-n-\overline{-} \\
& I j \cdot G j \cdot S j+\ldots I n \cdot G n \cdot S n
\end{aligned}
$$

La fórmula de peso extendida completa, a diferencia de la anterior, tiene en cuenta para la adicción, los dos lados de la ponderación a efectos de realizar la acumulación, es decir, que no se limita a sumar en la parte de la restricción sino que admite la posibilidad de acumular los principios constitucionales relevantes en el lado de la satisfacción. Luego, "todos los principios que jugaran en contra de la medida que se examina se opondrían en conjunto a todos los que jugaran a favor de ella"'.

Esta fórmula puede representarse de la siguiente manera (Carbonell, 2008, 30):

$$
\mathrm{Gi}^{\mathrm{m}}, \mathrm{j}^{\mathrm{n}}=\frac{\mathrm{Ii} \cdot \mathrm{Gi} \cdot \mathrm{Si}+\ldots \mathrm{I}^{\mathrm{m}} \cdot \mathrm{G}^{\mathrm{m}} \cdot \mathrm{S}^{\mathrm{m}}}{\mathrm{Ij} \cdot \mathrm{Gj} \cdot \overline{\mathrm{Sj}}+\ldots \mathrm{I}^{\mathrm{n}} \cdot \overline{\mathrm{G}^{\mathrm{n}} \cdot \mathrm{S}^{\mathrm{n}}}}
$$

Ahora bien, la pregunta que subyace a las anteriores representaciones es la relativa a que puede acumularse o "qué es aquello que es acumulable aditivamente". Tal cuestionamiento lo resuelve escuetamente Alexy al manifestar:

Los principios acumulados no pueden ser substancialmente redundantes. Sus objetos a optimizar deben ser materialmente diferentes. También tiene validez la regla según la cual la heterogeneidad es una condición de la acumulación

$9 \quad$ El derecho fundamental, cuya vulneración se examina, dejaría de ser entonces un guerrero aislado y se convertiría en el comandante de una tropa más o menos numerosa. 
aditiva (...) pueda fundarse en varios principios que no tienen ninguna intersección entre sí (Carbonell, 2008, 30).

\subsection{Critica a la fórmula del peso extendida}

El asunto sería simple si la heterogeneidad fuese un asunto de todo o nada. Con todo, este no es el caso. Los principios pueden intersectarse substancialmente. Es muy difícil determinar en cada caso completo si se está efectuando una acumulación adecuada y heterogénea de los principios referidos. Ello es más grave si se tiene en cuenta que es difícil precisar la naturaleza de los principios como para catalogarlos en los que resultan heterogéneos y los que no los son.

Las principales críticas a la fórmula del peso extendida podrían resumirse en tres. La primera refiere a que no está claro cómo determinar los principios que deben establecerse en cada uno de los extremos de la fórmula, al no existir parámetros que permitan identificar que se debe sumar contra que, o cuáles son las unidades que deben establecerse en la balanza. La segunda crítica refiere a que no está establecido la determinación de los aspectos que hacen que las unidades sean heterogéneas, no existe un claro criterio para demostrar lo homo o heterogéneo de la acumulación de los principios.

La tercera refiere a que la fórmula anotada no tiene en cuenta que los principios en conflicto pueden tener una relación o una cercanía diferente con el problema o con el caso debatido. Es decir, que en principio parece otorgarle las mismas implicaciones a los principios cercanos con aquellos que no lo sean. No establece una distinción entre los valores otorgados a los diferentes principios ni las relaciones de cercanía que puedan surgir entre ellos.

\subsection{Principio de la solución y presupuestos del principio de proporcionalidad sistémico}

En este escrito se pretenden bosquejar los elementos generales del principio de proporcionalidad, mediante los cuales se desea entender de manera sistémica el ordenamiento jurídico colombiano y explicar con mayor potencial comprensivo la toma de decisiones de la Corte Constitucional en el control de la ley. Para tal efecto se describen a continuación los presupuestos y la estructura del principio de proporcionalidad sistémico.

A los presupuestos referidos con anterioridad, es decir, que los derechos fundamentales son principios, que necesitan ser optimizados, que pueden ser restringidos, que tienen dos contenidos, hay que agregar tres elementos que permiten desarrollar el principio de proporcionalidad sistémico.

El primero refiere a que los principios están interconectados entre sí y ostentan relaciones complejas y variables. El entender al ordenamiento jurídico como sistema excluye en principio la idea de que en un conflicto de derechos fundamentales sólo 
dos de estos se puedan ver afectados. La característica sistémica del ordenamiento conlleva a que en un conflicto jurídico sean varios los principios jurídicos que resulten afectados, no obstante, que la afectación se dé en diferentes grados. Pensar que en los conflictos determinados solo dos principios jurídicos resultan afectados conllevaría a negar la característica sistémica del ordenamiento jurídico.

El segundo presupuesto afirma que los principios jurídicos afectados en un conflicto pueden serlo en diferentes grados, de conformidad con su cercanía o distancia con el problema. Por tanto, puede decirse que en un conflicto jurídico pueden existir principios afectados en diferentes grados; más afectados y menos afectados, dependiendo de la escala con la que se desee trabajar.

El tercer presupuesto establece que el principio de proporcionalidad reconoce que los resultados que entrega son siempre provisionales, que constituyen una respuesta plausible. No definitiva ni única, sino, simplemente un buen resultado argumentativo.

Entendidos de esta forma los presupuestos de la proporcionalidad se hace necesario explicar de manera un poco diferente los subprincipios que lo conforman. La forma de entender los subprincipios ostentan las variaciones estructurales de las premisas referidas.

\section{4. Estructura hipotética del principio de proporcionalidad sistémico}

El subprincipio de idoneidad ha de entenderse de una forma diferente. No pude decirse que en principio una medida legislativa coadyuva a satisfacer únicamente un fin constitucional. En la concepción clásica, el subprincipio de idoneidad es teleológico y unidimensional. Pues, requiere que las intervenciones en los derechos fundamentales deban ser adecuadas para contribuir a la obtención de un fin constitucionalmente legítimo; se dividía en dos partes: "la legitimidad constitucional del objetivo y la adecuación de la medida examinada" (Bernal, 2009, 28). Bajo la concepción que se pretende plantear, no puede decirse, si se quiere responder a la concepción sistémica del ordenamiento jurídico y a las prácticas reales de la Corte, que una intervención pretenda satisfacer a un principio constitucional, sino que debe entenderse que satisface varios principios constitucionales.

No obstante, que pueda darse el caso según el cual determinada medida satisface más a un principio que a otros, no puede decirse por ello que no se cumplen diversas finalidades con la medida. Ello se debe principalmente a que es un presupuesto lógico trascendental las diferentes consecuencias de la implementación de un elemento en un sistema. La prohibición de fumar, por ejemplo, puede proteger, además de la salud, el medio ambiente, el presupuesto estatal, la educación, la cultura.

Esta manera de plantear la existencia de la pluralidad de fines conlleva a un asunto de enorme importancia en la segunda parte de este subprincipio. Pues, la adecuación de la medida examinada para el cumplimiento "del fin" deberá ser observada no sólo desde el punto de vista unidimensional, sino también de 
conformidad con la adecuación de los diferentes fines. Lo cual aumenta en grado sumo la complejidad del procedimiento.

Las variaciones en la adecuación se implican en el subprincipio de necesidad. Según la fórmula clásica de la necesidad, "toda medida de intervención en los derechos fundamentales debe ser la más benigna con el derecho intervenido, entre todas aquellas que revisten por lo menos la misma idoneidad para contribuir a alcanzar el objetivo propuesto". Ahora, de conformidad con los presupuestos esbozados, se hace necesario revisar que la medida tomada es la más benigna con los derechos intervenidos; no sólo se hace necesario que sea la más benigna con uno de los derechos intervenidos sino que su consideración debe ser más sistémica. Los problemas que presenta esta concepción son muy variados, no obstante no serán objeto de este modesto escrito.

De conformidad con el subprincipio de proporcionalidad en sentido estricto, "las ventajas que se obtienen mediante la intervención en los principios constitucionales deben compensar los sacrificios que ésta implica para sus titulares y para la sociedad en general" (Bernal, 2003, 30). La ponderación, de conformidad con los que se ha dicho, deberá efectuarse desde el punto de vista que existe un número plural de principios constitucionales en juego, que se contraponen en todos los casos, a otro conjunto de principios.

Así pues, la manera clásica como se han entendido los subprincipios de la proporcionalidad debe mostrarse de una manera diferente. No se trata sólo de establecer la pluralidad de los elementos que conforman los dos lados de la balanza sino la cantidad de problemas que ello presenta.

\subsection{Delimitación de los dos lados de la balanza}

Los principales temas a los cuales se va a referir esta última parte del escrito son relativos a la determinación los derechos que deben ponderarse en cada lado de la balanza. Es decir, como se puede bosquejar una idea general para determinar, a manera de hipótesis, cuáles deben ser los principios que se deben tener en cuenta para obtener una agrupación adecuada que permita la aplicación del tercer subprincipio. La hipótesis que se sostiene es que se deben agrupar cuatro clases de principios en cada lado, según se refieran a los siguientes campos: derechos de defensa, derechos democráticos, principio de igualdad material, división de poderes; los cuales son, los ejes centrales del ordenamiento jurídico. Ello está soportado en dos líneas argumentativas: una histórica y otra de carácter inferencial.

Estas concepciones subyacen una forma de entender la Constitución. Según como se entienda la constitución va a surgir la hipótesis de los principios que deben tenerse en cuenta para aplicar la proporcionalidad en sentido estricto. Para establecer las ideas generales constitutivas de la Constitución se parte de la concepción histórica de la que surge de manera remota y se especifican las cualidades que han venido sumándosele con el tiempo. 
La historia remota de nuestra Constitución puede resaltarse con los movimientos jurídicos de la Francia revolucionaria. De conformidad con nuestra herencia obtenida de la familia jurídica romano germana, existen dos ejes generales que subyacen la idea de la Constitución: las garantías fundamentales y la división de poderes. Estos dos ejes generales se extraen del artículo 16 de la Declaración de 1789: "Toda sociedad en la cual no esté establecida la garantía de los derechos, ni determinada la separación de los poderes, carece de Constitución".

Sin embargo, se hace importante actualizar un poco dichos enfoques por dos argumentos generales. Se han dado variaciones a la concepción del constitucionalismo clásico; especialmente, por las modificaciones del constitucionalismo democrático, social y actual. Y de la práctica de la Corte Constitucional, se pueden inferir cierto grupo de argumentos que pueden categorizarse como campos recurrentes de fundamentación de las decisiones judiciales. Estas dos líneas argumentativas se explican sucintamente a continuación.

En un principio, de conformidad como se refirió en la época liberal burguesa, los principales elementos de la Constitución estaban referidos por la libertad (los derechos civiles y políticos) y la división de poderes, a los cuales, en esta nueva concepción, se les agrega la libertad política, la igualdad material y la separación de poderes (esta última como modificación de la concepción de división de poderes). Los derechos de defensa se entendían como un espacio vedado al poder central, en el cual los particulares eran libres de actuar de conformidad con su arbitrio. A esta clásica concepción de derechos, se hace necesario agregarle los principios y derechos democráticos, derivados del constitucionalismo democrático. Donde se superpone a la esfera de la libertad la facultad de participar en la conformación y toma de decisiones del Estado. Posteriormente, con el constitucionalismo social, se hace importante el principio de igualdad material ${ }^{10}$. Las desventajas sociales necesitaron ser reparadas mediante la eliminación de sus efectos en aras de buscar una discriminación inversa. En el constitucionalismo actual, dichos pilares han tomado una importancia sin precedentes, principalmente, desde la perspectiva de su normatividad. Queda por agregar que en el constitucionalismo actual la doctrina continental de la división de poderes ha sido superada por la de separación de poderes. A diferencia de la concepción de otrora, la separación implica la concepción según la cual se debilita el poder descomponiéndolo y haciendo que el mismo se controle; esta concepción ha sido comúnmente conocida como la noción de pesos y contrapesos ${ }^{11}$.

Según con la segunda línea argumentativa, los derechos de defensa, los derechos políticos, el principio de igualdad material, y la separación de poderes, son los campos

10 La concepción actual del ordenamiento jurídico agrega la existencia de derechos prestacionales a favor de las personas asociadas. A los derechos de defensa se les debe agregar la concepción de derechos prestacionales como otro de los elementos que definen el ordenamiento jurídico.

11 Esta es la concepción de origen anglosajón que también ha sido vista como aportada por la Constitución política en su artículo y desarrollada por la Corte Constitucional. 
argumentativos utilizados más recurrentes utilizados por la Corte Constitucional en el control de constitucionalidad de la ley. Es decir, al mejor estilo de la analogia iuris, se toman unos casos particulares los cuales se elevan a un amplio margen de abstracción y se establecen ciertos elementos esenciales. Distintas sentencias de la Corte dan cuenta de este fenómeno, con lo cual, se desestima en la práctica que la proporcionalidad en sentido estricto, conlleve sólo la comparación de dos principios constitucionales. Y muestran como los cuatro puntos cardinales referidos son una importante hipótesis a evaluar. Hipótesis que, por demás, está muy emparentada con la dignidad humana como fundamento del orden jurídico.

La Corte Constitucional en la parte sustancial ha afirmado que la dignidad humana pueden derivarse tres líneas generales: vivir como se quiere vivir bien, y vivir sin humillaciones. Según la sentencia de la Corte Constitucional T 881 de 2002, existen respecto de la dignidad humana "tres lineamientos claros y diferenciables: (i) La dignidad humana entendida como autonomía o como posibilidad de diseñar un plan vital y de determinarse según sus características (vivir como quiera). (ii) La dignidad humana entendida como ciertas condiciones materiales concretas de existencia (vivir bien). Y (iii) la dignidad humana entendida como intangibilidad de los bienes no patrimoniales, integridad física e integridad moral (vivir sin humillaciones)". De forma general las tres líneas referidas son similares a: derechos autonómicos o democráticos, de igualdad material y de defensa.

En el caso de la Sentencia C 141 de 2010, unos de los argumentos utilizados para declarar inconstitucional la medida revisada, fueron: la alternancia en el poder (principio democrático), la generalidad de las leyes (igualdad), la descompensación de los órganos de control (división de poderes). En la sentencia C 673 de 2001, los cargos del actor se fundamentan - básicamente en dos grupos de argumentos: "por un lado, la violación del principio de igualdad (Art. 13 C.P.) y, por otro, la violación de diversos derechos de libertad - en este caso el libre desarrollo de la personalidad, la libertad de expresión, enseñanza y cátedra".

Los cuatro campos referidos son tan importantes que su afectación grave conlleva la sustitución de la Constitución. En la sentencia C 588 de 2009, se estimó que existía sustitución de la constitución porque se afectaba de manera grave "el principio de separación de poderes", "la pretensión de universalidad de las reglas" (igualdad), "interrupción del principio del mérito y del mecanismo del concurso público" (principios democráticos), "la vigencia del artículo 40-72” (principio democrático) "y lo propio cabe aseverar del derecho a la igualdad que, durante idéntico lapso temporal, dejará de aplicarse a los mismos ciudadanos"12.

12 En un sentido similar pueden revisarse las sentencias de la Corte Constitucional C 309de 1997, C 836 de 2001, C 588 de 2009, C 141 de 2010. 


\section{CONCLUSIONES}

Pese a las múltiples conclusiones que pueden extraerse de la temática abordada, se extraerán cinco ideas generales sobre las cuales, pese a su fácil conocimiento, se desea insistir con especial vehemencia. Estas ideas son: la idiosincrasia de la recepción, los inconvenientes de su integración en el caso de la proporcionalidad, el alejamiento práctico de la proporcionalidad, la idea de revisar la forma como decide para explicar menor los métodos utilizados, y la necesidad de investigar a fondo la metodología jurídica.

Es conveniente resaltar la importancia de la recepción de teorías jurídicas. No puede verse este fenómeno como algo enteramente negativo. Ha sido una constante en la configuración del ordenamiento jurídico interno. No existe una fuerte construcción teórica y dogmática acerca del derecho en nuestro país, por tanto, ha sido la teoría y doctrina extranjera uno de los principales elementos de cambio y evolución del ordenamiento jurídico interno.

La recepción de dichas teorías a pesar que pueda argumentarse que no se tiene los ambientes hermenéuticos estándares para entenderlos o que no están diseñadas para nuestro ordenamiento jurídico, han constituido un aporte que ha sido tomado como interpretaciones idiosincrásicas o se ha hecho un acomodamiento jurídico para darles paso. Han demostrado funcionar más o menos de manera que existe la renovación o el dinamismo jurídico.

En el caso de la adopción del principio de proporcionalidad de origen, especialmente germano, la Corte Constitucional lo acomoda de manera idiosincrática con el juicio de igualdad de origen anglosajón ${ }^{13}$. Pese a reconocer su diferentes orígenes para la Corte estas metodologías no son contradictorias sino complementarias, y, a partir de allí plantea algo que ha denominado como el juicio integrado de proporcionalidad, que toma presupuestos de ambas metodologías y las combina.

No obstante, los esfuerzos desarrollados por la Corte para adoptar el juicio de proporcionalidad esta metodología está alejada de la forma en la que decide la Corte, pues, además de basarse en una concepción unidimensional del ordenamiento jurídico, apartada, como se mencionó, de la concepción sistémica, no da cuenta del proceso real de decisión de la Corte en el control de constitucionalidad de la leyes.

Luego, no puede decirse que la proporcionalidad sea en realidad la metodología utilizada, pues, la argumentación de la Corte en los juicios de proporcionalidad es sistemática. La forma como decide la Corte muestra una concepción de ordenamiento amplia y sistemática que se aleja de la forma clásica como ha querido explicarse el principio de proporcionalidad.

Así pues, la metodología jurídica está en un proceso de cambio de paradigma que tiende una invitación a profundizar en la investigación en estos temas; a tratar de

13 Especialmente ver Corte Constitucional Sentencia C 093 de 2001. 
solucionar los múltiples problemas que presenta la interpretación y aplicación de los principios constitucionales.

\section{REFERENCIAS}

Alexy, R. (2002). Teoría de los derechos fundamentales, Madrid: Centro de Estudios Políticos y Constitucionales, Impreso en Solana e hijos, A.G., S.A.

Alexy, R. (2004). El Concepto y la Validez del Derecho, Barcelona: Gedisa.

Alexy, R. (2004). Teoría del Discurso y los Derechos Humanos, Bogotá: Universidad Externado de Colombia. 2004.

Bernal, C. (2005). El Derecho de los derechos. Bogotá: Universidad Externado de Colombia.

Bernal, C. (2007). El Neoconstitucionalismo a debate. Bogotá: Universidad Externado.

Bernal, C. (2009). El Neoconstitucionalismo y la normatividad en el derecho. Bogotá: Universidad Externado de Colombia.

Bernal, C. (2003). El principio de proporcionalidad y los derechos fundamentales Madrid: Centro de Estudios Políticos y Constitucionales.

Carbonell, M. (2008). El Principio de proporcionalidad y la interpretación constitucional. Ministerio de Justicia y Derechos Humanos. Quito, Ecuador, 2008.

Corte Constitucional de Colombia. Sentencia C 093 de 2001. Magistrado ponente Alejandro Martínez Caballero. Sala plena. Enero 31 de 2001.

Declaración de los derechos del hombre y el ciudadano. 1789.

López, D. (2004). Teoría impura del derecho. La transformación de la cultura jurídica latinoamericana. Bogotá: Legis - Universidad de los Andes, Universidad Nacional.

López, D. (2006). Interpretación Constitucional. Bogotá: Universidad Nacional de Colombia. Segunda edición.

Stammler, R. (1923). Theorie der Rechtswissenschaft, Buchhandlung des Weiseshauses, Halle, 2a. Edición. 